\title{
The Nielsen coincidence theory on topological manifolds
}

\author{
by
}

Jerzy J e z i e r s ki (Warszawa)

\begin{abstract}
We generalize the coincidence semi-index introduced in [D-J] to pairs of maps between topological manifolds. This permits extending the Nielsen theory to this class of maps.
\end{abstract}

Introduction. In this paper we generalize the coincidence semi-index theory, introduced in $[\mathrm{D}-\mathrm{J}]$ in the smooth case, to pairs of maps between topological manifolds. It will be based on the topological transversality lemma (1.1). To show that this new theory generalizes the previous one it will be necessary to reformulate [D-J] since the graphs of any two maps are never topologically transverse. This is done in Section 2: we give three equivalent versions of the semi-index in the smooth case and then we show that one of them coincides with the semi-index defined in Section 1. In Section 3 we prove a Wecken type theorem on realizing the above Nielsen number.

1. The coincidence semi-index on topological manifolds. Throughout this paper we consider pairs of maps $f, g: M \rightarrow N$ such that $M, N$ are topological separable manifolds without boundary and the coincidence set $\Phi(f, g)=\{x \in M: f x=g x\}$ is compact. The construction of the semi-index we present is based on the transversality lemma (1.1) below.

Let $P \subset W$ and $V$ be topological manifolds and $\xi$ a normal microbundle of $P$ in $W$, i.e. the total space of $\xi$ is an open subset of $W$ containing $P$. Recall that a map $h: V \rightarrow W$ is called topologically transverse (briefly $t$-transverse) to $\xi$ if $h^{-1} P$ is a topological submanifold in $V$ admitting a normal microbundle $\nu$ such that for any $x \in h^{-1} P$ a neighbourhood of $x$ in $\nu_{x}$ is mapped by $h$ homeomorphically onto a neighbourhood of $h x$ in $\xi_{h x}[\mathrm{~K}-\mathrm{S}]$.

1991 Mathematics Subject Classification: Primary 55M20; Secondary 57N99. 
Lemma (1.1). Let $V, W$ be topological manifolds, and $P \subset W$ a submanifold with a normal microbundle $\xi$. Let $C, D \subset V$ be closed subsets and $h: V \rightarrow W$ a continuous map transverse to $\xi$ near $C$. Then there is a homotopy $\left\{h_{t}\right\}$ based in a given neighbourhood of $D-C$ such that $h_{0}=h$ and $h_{1}$ is transverse to $\xi$ near $C \cup D$. Moreover, if $d$ is a metric on $W$, and $\varepsilon: V \rightarrow(0, \infty)$ any function then we may require $d\left(h_{t}(x), h(x)\right)<\varepsilon(x)$ for any $x \in V$ and $0 \leq t \leq 1$.

Proof. Let $p, v, w$ denote the dimensions of $P, V, W$ respectively. For $v \neq 4 \neq w-p$ the assertion follows from Thm. (1.1) in Essay III of [K-S]. The assumption $v \neq 4$ is not necessary now since by [F] every 4-manifold is almost smoothable.

The case $w-p=4$ follows from Thm. (5.3) of $[\mathrm{Sc}]$ since by $[\mathrm{F}]$ the Rokhlin theorem fails in the TOP category.

It will be convenient to start with a more general situation. For given manifolds $V, W, P \subset W$ and a map $h: V \rightarrow W$ we define a relation on $h^{-1} P$ which will also be called the Nielsen relation: $x \simeq y$ iff there is a path $\omega$ joining these points in $V$ such that $h \omega$ is homotopic in $W$ to a path in $P$. ( Let $f, g: M \rightarrow N$ be a pair of maps: we put $h=(f, g): V=M \rightarrow$ $W=N \times N, P=\Delta N$. Then $\Phi(f, g)=h^{-1} \Delta N$ and the above relation coincides with the classical Nielsen relation [S], [D-J].) Moreover, we will always assume $h^{-1} P$ to be compact.

Now suppose that the dimensions of the manifolds $V, P \subset W$ satisfy $v+p=w, \xi$ is a normal microbundle of $P$ in $W$ and $h: V \rightarrow W$ a map t-transverse to $\xi$. Since $\operatorname{dim} h^{-1} P=v+p-w=0, h^{-1} P$ is discrete and $\nu$ splits into open sets, each projected into a point in $h^{-1} P$.

Let $\alpha, \beta$ denote two microbundles of the same dimension $r$ and let $\alpha_{x}$ denote the fibre over a point $x$ of the zero section of $\alpha$. Then any generator $z_{x} \in H_{r}\left(\alpha_{x}, \alpha_{x}-x\right)$ will be called a (local) orientation of the microbundle $\alpha$ at $x$. Let $k: \alpha \rightarrow \beta$ be a microbundle map such that the restriction $k_{\mid \alpha_{x}}$ is a homeomorphism near $x \in \alpha_{x}$. Then this restriction determines an orientation $\left(k_{\mid}\right)_{*} z_{x} \in H_{r}\left(\beta_{k x}, \beta_{k x}-k x\right)$ which by abuse of notation will also be denoted by $k_{*} z_{x}$.

Let $\omega$ be a path establishing the Nielsen relation between $x, y \in h^{-1} P$. Let $\gamma_{0} \in H_{v}(V, V-x)$ be an orientation of the manifold $V$ at $x$. Then $h_{*} \gamma_{0} \in H_{v}\left(\xi_{h x}, \xi_{h x}-h x\right)$ is an orientation of the microbundle $\xi$ at $h x \in P$. Let $\gamma_{t}$ denote the translation of $\gamma_{0}$ along $\omega(t)$.

Definition (1.2). We will say that two points $x, y \in h^{-1} P$ are $R$-related $(x R y)$ iff there is a path $\omega$ establishing the Nielsen relation between them such that the translation of the orientation $h_{*} \gamma_{0}$ along a path in $P$ homotopic to $h \omega$ in $W$ is opposite to $h_{*} \gamma_{1}$. 
Let $A \subset h^{-1} P$ be a subset. It can be represented as $A=\left\{a_{1}, b_{1}, \ldots\right.$, $\left.a_{k}, b_{k}: c_{1}, \ldots, c_{s}\right\}$ where $a_{i} R b_{i}$ for any $i$ and $c_{i} R c_{j}$ for no $i \neq j$. We will call the elements $\left\{c_{1}, \ldots, c_{s}\right\}$ free in this decomposition.

LEMma (1.3). The number of free elements does not depend on the decomposition of $A$.

Proof. See the proof of (1.3) in [D-J].

We define the semi-index of the set $A \subset h^{-1} P$ with respect to $\xi$ to be the number of free elements in any decomposition of $A$, and we denote this number by $\mid$ ind $\left.\right|_{\mathrm{t}}(h, \xi: A)$.

Lemma (1.4) (Homotopy invariance). Let $H: V \times[0,1] \rightarrow W$ be a map $t$-transverse to a normal microbundle $\xi$ of $P$ in $W(v+p=w)$. Let $A$ be a clopen subset of $H^{-1} P$ and set $A_{t}=\{x \in V:(x, t) \in A\}, H_{t}(x)=H(x, t)$ for $0 \leq t \leq 1$. Then $\mid$ ind $\left.\right|_{\mathrm{t}}\left(H_{0}, \xi: A_{0}\right)=\mid$ ind $\left.\right|_{\mathrm{t}}\left(H_{1}, \xi: A_{1}\right)$.

Proof. By transversality, $H^{-1} P$ is a one-dimensional manifold. Consider first a connected component of $H^{-1} P$ with ends $(x, 0),\left(x^{\prime}, 0\right)$. We will show that $x, x^{\prime} \in H_{0}^{-1} P$ are $R$-related. Then we will show that if $(x, 0),(y, 1)$ are ends of a connected component and $\left(x^{\prime}, 0\right),\left(y^{\prime}, 1\right)$ are ends of another one then $x, x^{\prime} \in H_{0}^{-1} P$ are $R$-related iff $y, y^{\prime} \in H_{1}^{-1} P$ are $R$-related. The above facts show that there are decompositions of $A_{0}$ and $A_{1}$ with the same number of free points, which proves our lemma.

Let $\nu$ be a normal microbundle over $H^{-1} P$ from the transversality assumption. Consider a connected component $A$ with ends $(x, 0),(y, 0) \in$ $V \times 0$. We will show that $x R y$ in $H_{0}^{-1} P$.

Let $\omega(t)=\left(\omega_{1}(t), \omega_{2}(t)\right) \subset V \times I$ be a parametrization of this component. Then $(t, s) \rightarrow H\left(\omega_{1}(t), s \omega_{2}(t)\right)$ is a homotopy between $H\left(\omega_{1}(t), 0\right)=H_{0} \omega_{1}$ and the path $H \omega \subset P$. Now $\omega_{1}$ establishes the Nielsen relation between $x=\omega_{1}(0)$ and $y=\omega_{1}(1)$ in $H_{0}^{-1} P$.

We show that $\omega_{1}$ also establishes the $R$-relation. Let $\gamma_{0}$ be a local orientation of the manifold $M \times 0$ at $(x, 0)$ and let $\gamma_{t}$ be its translation along the path $\left(\omega_{1}, 0\right)$. Then $\gamma_{0}$ is also an orientation of the microbundle $\nu$ at $(x, 0)$. Let $\widehat{\gamma}_{t}$ denote its translation along $\omega$. Notice that then $\widehat{\gamma}_{1}=-\gamma_{1}$. On the other hand, $H_{*} \widehat{\gamma}_{t}$ is the translation of the orientation of $\xi$ along the path $H \omega$ (which is contained in $P$ and homotopic to $H_{0} \omega_{1}$ in $N$ ). For $t=1$ we obtain $H_{*} \widehat{\gamma}_{1}=H_{0 *} \widehat{\gamma}_{1}=-H_{0 *} \gamma_{1}$, proving $x R y$ in $H_{0}^{-1} P$.

Now we consider the second case: let $\omega(t)=\left(\omega_{1}(t), \omega_{2}(t)\right), \omega^{\prime}(t)=$ $\left(\omega_{1}^{\prime}(t), \omega_{2}^{\prime}(t)\right)$ be parametrizations of the two components with ends $\omega(0)=$ $(x, 0), \omega(1)=(y, 1), \omega^{\prime}(0)=\left(x^{\prime}, 0\right), \omega^{\prime}(1)=\left(y^{\prime}, 1\right)$. Suppose that a path $u \subset V$ establishes the $R$-relation between $x, x^{\prime} \in H_{0}^{-1} P$. We will show that $-\omega_{1}+u+\omega_{1}^{\prime}$ establishes the $R$-relation between $y, y^{\prime} \in H_{1}^{-1} P$. Let $\bar{u}$ denote 
a path in $P$ homotopic in $W$ to $H_{0} u$. Since

$$
H_{1}\left(-\omega_{1}+u+\omega_{1}^{\prime}\right) \simeq H\left(-\omega+(u, 0)+\omega^{\prime}\right) \simeq H(-\omega)+\bar{u}+H \omega^{\prime} \subset P,
$$

$-\omega_{1}+u+\omega_{1}^{\prime}$ establishes the Nielsen relation between $y, y^{\prime} \in H^{-1} P$.

Let $\gamma_{1}$ be an orientation of $M \times 1$ at $(y, 1)$. It is also an orientation of the microbundle $\nu$ at $(y, 1)=\omega(1)$; let $\gamma_{t}$ be its translation along $\omega(t)$. Then $\gamma_{0}$ is an orientation of $M \times 0$ at $(x, 0)$; let $\gamma_{t}^{\prime}$ be its translation along $(u, 0)$ to $\left(x^{\prime}, 0\right)$. Then $\gamma_{1}^{\prime}$ is an orientation of $\nu$ at $\left(x^{\prime}, 0\right)=\omega^{\prime}(0)$; let $\gamma_{t}^{\prime \prime}$ be its translation along $\omega^{\prime}$. Notice that $\gamma_{1}^{\prime \prime}$ is the translation of the orientation $\gamma_{1}$ of $M \times 1$ along $-\omega_{1}+u+\omega_{1}^{\prime}$.

It remains to show that $H_{1 *} \gamma_{1}^{\prime \prime}$ is opposite to the translation of the orientation $H_{1 *} \gamma_{1}$ of $\xi$ along a path lying in $P$ and homotopic in $W$ to $H_{1}\left(-\omega_{1}+u+\omega_{1}^{\prime}\right)$. Notice that $H(-\omega)+\bar{u}+H \omega^{\prime}$ is such a path. Now

- the translation of $H_{*} \gamma_{1}$ along $-H \omega$ gives $H_{*} \gamma_{0}=H_{0 *} \gamma_{0}$,

- the translation of $H_{0 *} \gamma_{0}$ along $\bar{u}$ gives $-H_{0 *} \gamma_{0}^{\prime}=-H_{*} \gamma_{0}^{\prime \prime}$ since $x R x^{\prime}$,

- the translation of $-H_{*} \gamma^{\prime \prime}$ along $H \omega^{\prime}$ gives $-H_{*} \gamma_{1}^{\prime \prime}=-H_{1 *} \gamma_{1}^{\prime \prime}$

hence we obtain the orientation opposite to $H_{1 *} \gamma_{1}^{\prime \prime}$, which proves $y R y^{\prime}$ in $H_{1}^{-1} P$.

Let $A_{0}$ from the above lemma be a Nielsen class of $H_{0}$. Then $A_{1}$ is contained in a Nielsen class $A_{1}^{\prime}$ of $H_{1}$ and $A_{1}^{\prime}-A_{1}$ is the union of pairs of points where each pair is the boundary of a component of $H^{-1} P$. Thus $A_{1}^{\prime}-A_{1}$ splits into pairs of $R$-related points, which implies that the semiindices of $A_{0}$ and $A_{1}$ are the same. Since by (1.1) any homotopy between transverse maps may be deformed to a transverse homotopy, we obtain:

Lemma (1.5). Let $H: V \times I \rightarrow W$ be a $\Phi$-compact homotopy between the maps $H_{0}, H_{1}: V \rightarrow W$ transverse to a normal bundle $\xi$ of $P$ in $W$. Let $A_{i} \subset H_{i}^{-1} P$ be Nielsen classes corresponding under this homotopy $(i=0,1)$. Then

$$
\mid \text { ind }\left.\right|_{\mathrm{t}}\left(H_{0}, \xi: A_{0}\right)=|\operatorname{ind}|_{\mathrm{t}}\left(H_{1}, \xi: A_{1}\right) .
$$

A Nielsen class $A$ of a t-transverse map $h$ will be called essential if |ind $\left.\right|_{\mathrm{t}}(h, \xi: A) \neq 0$. Define the Nielsen number $N(h)$ of $h$ to be the number of essential classes; in fact, $N(h)$ also depends on $P$ and $\xi$. By Lemma (1.5), $N(h)$ is a correctly defined $\Phi$-homotopy invariant. This definition implies $\# h^{-1} P \geq N(h)$.

Let $h: V \rightarrow W$ be a $\Phi$-compact map (not necessarily transverse). We define $N(h)=N\left(h^{\prime}\right)$ where $h^{\prime}$ is any transverse map $\Phi$-compactly homotopic to $h$. By $(1.5)$ this is a correctly defined $\Phi$-compact homotopy invariant.

Theorem (1.6). Let $h: V \rightarrow W$ be $\Phi$-compact. Then $h^{-1} P$ contains at least $N(h)$ points. 
Proof. This is evident when $h$ is transverse to $P$. In the general case suppose that $\# h^{-1} P=k<N(h)$. For any $x \in h^{-1} P$ take a contractible neighbourhood $W_{h x}$ of $h x$ in $W$ such that $W_{h x} \cap P$ is a deformation retract of $W_{h x}$. Then take a neighbourhood $V_{x}$ of $x$ in $V$ such that $h\left(\operatorname{cl} V_{x}\right) \subset W_{h x}$. Let $\left\{h_{t}\right\}$ be a compact homotopy, supported in $\bigcup\left\{V_{x}: x \in h^{-1} P\right\}$, from $h$ to a map t-transverse to $\xi$. Since this homotopy may be arbitrarily small, we may assume $h_{t}\left(V_{x}\right) \subset W_{h x}$ hence $h_{1}^{-1} P \cap V_{x}$ belongs to a Nielsen class. Now $h_{1}^{-1} P$ contains at most $k$ nonempty Nielsen classes, contradicting $N\left(h_{1}\right)=$ $N(h)>k$.

Now we may apply the above results to coincidences: let $f, g: M \rightarrow$ $N$ be a $\Phi$-compact map of $n$-manifolds. We put $V=M, W=N \times N$, $h(x)=(f x, g x), P=\Delta N$ and $\xi$ the microbundle $N \times N \ni(x, y) \rightarrow$ $(x, x) \in \Delta N$. We define the coincidence Nielsen number of the pair $f, g$ by $N(f, g)=N(h)$. Since $\Phi(f, g)=h^{-1}(\Delta N)$ and both Nielsen relations coincide, Thm. (1.6) implies

Corollary (1.7). $N(f, g)$ is a $\Phi$-compact homotopy invariant and $\Phi(f, g)$ contains at least $N(f, g)$ points.

Next we show that in the oriented case our semi-index equals the absolute value of the ordinary coincidence index $[\mathrm{S}],[\mathrm{V}],[\mathrm{D}-\mathrm{K}]$.

Lemma (1.8). Let $f, g: M \rightarrow N$ be a $\Phi$-compact pair, $M, N$ oriented manifolds, and $A \subset \Phi(f, g)$ a Nielsen class. Then

$$
|\operatorname{ind}(f, g: A)|=|\operatorname{ind}|_{\mathrm{t}}(f, g: A) \text {. }
$$

Proof. Since both ind and $\mid$ ind $\left.\right|_{\mathrm{t}}$ are $\Phi$-homotopy invariant, we may assume that the pair $f, g$ is transverse. Consider $x_{0}, x_{1} \in \Phi(f, g)$. We will show that $x_{0} R x_{1}$ iff $\operatorname{ind}\left(f, g: x_{0}\right)=-\operatorname{ind}\left(f, g: x_{1}\right)$. Let $u$ be a path establishing the Nielsen relation between $x_{0}$ and $x_{1}$. We notice that in the oriented case the translation of a local orientation $\alpha_{0} \in H_{n}\left(M, M-x_{0}\right)$ along $u$ gives $\alpha_{1} \in H_{n}\left(M, M-x_{1}\right)$ such that both $\alpha_{0}, \alpha_{1}$ determine the same global orientation $\alpha \in H_{n} M$. Similarly, the translation of a generator $\beta_{0} \in H_{n}\left(y_{0} \times\left(N, N-y_{0}\right)\right)$ (along a path in $\Delta N$ from $\left(y_{0}, y_{0}\right)$ to $\left(y_{1}, y_{1}\right)$ ) gives $\beta_{1} \in H_{n}\left(y_{1} \times\left(N, N-y_{0}\right)\right)$ such that $\beta_{0}$ and $\beta_{1}$ correspond to the same generator $\beta \in H_{n}(N \times N, N \times N-\Delta N)$. Thus the points $x_{0}, x_{1}$ are $R$-related iff the fixed global orientations are preserved by the pair $f, g$ at one of these points and reversed at the other. But the last means exactly $\operatorname{ind}\left(f, g: x_{0}\right)=-\operatorname{ind}\left(f, g: x_{1}\right)$ as claimed.

2. The semi-index on smooth manifolds. In $[\mathrm{D}-\mathrm{J}]$ we considered pairs of maps $f, g: M \rightarrow N$ between two smooth closed $n$-manifolds. For each Nielsen class we defined a semi-index which we will denote here by $\mid$ ind $\left.\right|_{\mathrm{d}}$. To do this we replaced the given pair by a transverse one (in the 
smooth category), here called a $d$-transverse pair, and we defined on $\Phi(f, g)$ a reducibility relation (here termed the $R_{\mathrm{d}}$-relation). Then any Nielsen class $A \subset \Phi(f, g)$ was represented as $A=\left\{a_{1}, b_{1}, \ldots, a_{k}, b_{k} ; c_{1}, \ldots, c_{s}\right\}$ where $a_{i} R_{\mathrm{d}} b_{i}$ for any $i=1, \ldots, k$, but $c_{i} R_{\mathrm{d}} c_{j}$ for no $i, j=1, \ldots, s, i \neq j$, and we defined $|\operatorname{ind}|_{\mathrm{d}}(f, g: A)=s$. However, the approach from [D-J] is not convenient for comparison with the method from Section 1 of the present paper: in [D-J] we considered transversality in $M \times N$, while here in $N \times N$. To overcome this difference we will give three equivalent versions $(\mathrm{A}),(\mathrm{B})$, (C), of d-transversality and of the $R_{\mathrm{d}}$-relation. Method (A) is the easiest to formulate: it does not involve any product spaces. Method (B) is the one given in $[\mathrm{D}-\mathrm{J}]$. We will show that in the smooth case, $\mid$ ind $\left.\right|_{\mathrm{t}}$ coincides with $\mid$ ind $\left.\right|_{\mathrm{d}}$ obtained by using method $(\mathrm{C})$. Then the equivalence of $(\mathrm{B})$ and $(\mathrm{C})$ implies the desired equality $\mid$ ind $\left.\right|_{\mathrm{d}}=\mid$ ind $\left.\right|_{\mathrm{t}}$.

Consider a $\Phi$-compact, smooth pair of maps $f, g: M \rightarrow N$ between smooth $n$-manifolds. Then for any $x \in \Phi(f, g)$ the following three conditions are equivalent:

(A) The difference of the tangent homomorphisms $f_{*}-g_{*}: T_{x} M \rightarrow T_{f x} N$ is an isomorphism.

(B) Let $\Gamma_{f}=\{(x, y) \in M \times N: y=f x\}$ denote the graph of $f$. Then the tangent spaces $T_{(x, f x)} \Gamma_{f}, T_{(x, g x)} \Gamma_{g}$ span the whole $T_{(x, f x)} M \times N$.

(C) The intersection of the subspaces $\operatorname{im}(f, g)_{*}$ and $T_{(f x, g x)} \Delta N$ in $T_{(f x, g x)} N \times N$ is zero.

Notice that (B) means that the pair $f, g$ is transverse in the sense of [D-J]; here we will call it d-transverse. Assume that $f, g: M \rightarrow N$ is d-transverse and $\omega$ is a path establishing the Nielsen relation between the points $x, y \in$ $\Phi(f, g)$. Fix an ordered basis $a_{1}^{0}, \ldots, a_{n}^{0}$ of $T_{x} M$ and let $a_{1}^{t}, \ldots, a_{n}^{t} \in T_{r(t)} M$ be its translation along $\omega$. Then

$\left(\mathrm{A}^{\prime}\right)\left(f_{*}-g_{*}\right) a_{1}^{0}, \ldots,\left(f_{*}-g_{*}\right) a_{n}^{0}$ form a basis of $T_{f x} N$, $\left(f_{*}-g_{*}\right) a_{1}^{1}, \ldots,\left(f_{*}-g_{*}\right) a_{n}^{1}$ form a basis of $T_{g x} N$.

Let $\alpha_{0}$ and $\alpha_{1}$ denote the orientations determined by these bases.

$\left(\mathrm{B}^{\prime}\right)\left(a_{1}^{t}, f_{*} a_{1}^{t}\right), \ldots,\left(a_{n}^{t}, f_{*} a_{n}^{t}\right)$ form a basis of $T_{(\omega(t), f \omega(t))} \Gamma_{f}$, $\left(a_{1}^{t}, g_{*} a_{1}^{t}\right), \ldots,\left(a_{n}^{t}, g_{*} a_{n}^{t}\right)$ form a basis of $T_{(\omega(t), g \omega(t))} \Gamma_{g}$.

Let $\beta_{t}(f)$ and $\beta_{t}(g)$ denote the orientations determined by these bases. Let $\beta_{0}=\beta_{0}(f) \wedge \beta_{0}(g)$ and $\beta_{1}=\beta_{1}(f) \wedge \beta_{1}(g)$.

$\left(\mathrm{C}^{\prime}\right)\left(f_{*} a_{1}^{0}, g_{*} a_{1}^{0}\right), \ldots,\left(f_{*} a_{n}^{0}, g_{*} a_{n}^{0}\right)$ form a basis of the fibre of the normal bundle $\nu=T(N \times N) / T(\Delta N)$ of $\Delta N \subset N \times N$ at the point $(f x, g x)$, and $\left(f_{*} a_{1}^{1}, g_{*} a_{1}^{1}\right), \ldots,\left(f_{*} a_{n}^{1}, g_{*} a_{n}^{1}\right)$ form a basis of the fibre of $\nu$ at $(f y, g y)$. 
Let $\gamma_{0}, \gamma_{1}$ denote the orientations determined by these bases.

THEOREM (2.1). Under the above notations the following three conditions are equivalent:

$\left(\mathrm{A}^{\prime \prime}\right) \alpha_{1}$ is opposite to the translation of $\alpha_{0}$ along $f \omega \subset N$.

$\left(\mathrm{B}^{\prime \prime}\right) \beta_{1}$ is opposite to the translation of $\beta_{0}$ along $(\omega, f \omega) \subset M \times N$.

$\left(\mathrm{C}^{\prime \prime}\right) \gamma_{1}$ is opposite to the translation of $\gamma_{0}$ along $(f \omega, f \omega) \subset N \times N$.

Proof. The proof will be based on the following

Lemma (2.2). Let $V, W$ be $n$-dimensional real linear spaces, $a_{1}, \ldots, a_{n}$, $a$ basis of $V$ and $b_{1}, \ldots, b_{n}, c_{1}, \ldots, c_{n} \in W$ such that $b_{1}-c_{1}, \ldots, b_{n}-c_{n}$ is a basis of $W$. Then the orientations of $V \times W$ given by the ordered bases $\left(a_{1}, b_{1}\right), \ldots,\left(a_{n}, b_{n}\right),\left(a_{1}, c_{1}\right), \ldots,\left(a_{n}, c_{n}\right)$ and $\left(0, b_{1}-c_{1}\right), \ldots,\left(0, b_{n}-c_{n}\right)$, $\left(a_{1}, 0\right), \ldots,\left(a_{n}, 0\right)$ coincide.

Pr o of. Consider the homotopies

$$
t \rightarrow\left(a_{1}, b_{1}-t c_{1}\right), \ldots,\left(a_{n}, b_{n}-t c_{n}\right),\left(a_{1},(1-t) c_{1}\right), \ldots,\left(a_{n},(1-t) c_{n}\right)
$$

and

$$
t \rightarrow\left(t a_{1}, b_{1}-c_{1}\right), \ldots,\left(t a_{n}, b_{n}-c_{n}\right),\left(a_{1}, 0\right), \ldots,\left(a_{n}, 0\right)
$$

in the Stiefel space of ordered bases.

Proof of Theorem (2.1). $\left(\mathrm{A}^{\prime \prime}\right) \Leftrightarrow\left(\mathrm{B}^{\prime \prime}\right)$. We compare $\beta_{1}$ and the translation of $\beta_{0}$. The orientation $\beta_{1}$ is given by the basis

$$
\left(a_{1}^{1}, f_{\#} a_{1}^{1}\right), \ldots,\left(a_{n}^{1}, f_{\#} a_{n}^{1}\right),\left(a_{1}^{1}, g_{\#} a_{1}^{1}\right), \ldots,\left(a_{n}^{1}, g_{\#} a_{n}^{1}\right),
$$

which by $(2.2)$ is equivalent to

$$
\left(0,\left(f_{\#}-g_{\#}\right) a_{1}^{1}\right), \ldots,\left(0,\left(f_{\#}-g_{\#}\right) a_{n}^{1}\right),\left(a_{1}^{1}, 0\right), \ldots,\left(a_{n}^{1}, 0\right) .
$$

For the same reasons $\beta_{0}$ is equivalent to

$$
\left(0,\left(f_{\#}-g_{\#}\right) a_{1}^{0}\right), \ldots,\left(0,\left(f_{\#}-g_{\#}\right) a_{n}^{0}\right),\left(a_{1}^{0}, 0\right), \ldots,\left(a_{n}^{0}, 0\right) .
$$

Now let $\left(0, b_{1}^{t}\right), \ldots,\left(0, b_{n}^{t}\right),\left(a_{1}^{t}, 0\right), \ldots,\left(a_{n}^{t}, 0\right)$ denote the translation of the last basis along $(\omega, f \omega)$ in $M \times N$. For $t=1$ we obtain $\left(0, b_{1}^{1}\right), \ldots,\left(0, b_{n}^{1}\right)$, $\left(a_{1}^{1}, 0\right), \ldots,\left(a_{n}^{1}, 0\right)$, which agrees with $\beta_{1}$ iff the orientations of $T_{f y} N$ given by the bases $\left(f_{\#}-g_{\#}\right) a_{1}^{1}, \ldots,\left(f_{\#}-g_{\#}\right) a_{n}^{1}$ and $b_{1}^{1}, \ldots, b_{n}^{1}$ are equal, i.e. $\alpha_{1}$ equals the translation of $\alpha_{0}$.

$\left(\mathrm{A}^{\prime \prime}\right) \Leftrightarrow\left(\mathrm{C}^{\prime \prime}\right)$. We compare $\gamma_{1}$ and the translation of $\gamma_{0}$ along $(f \omega, f \omega)$. The homotopy $t \rightarrow\left(\ldots,\left(f_{\#} a_{i}^{0}-t g_{\#} a_{i}^{0},(1-t) g_{\#} a_{i}^{0}\right), \ldots\right)$ shows that $\gamma_{0}$ may also be represented by $\left(b_{1}^{0}, 0\right), \ldots,\left(b_{n}^{0}, 0\right)$ where $b_{1}^{0}, \ldots, b_{n}^{0}$ represents $\alpha_{0}$. Let $b_{1}^{t}, \ldots, b_{n}^{t}$ denote again the translation of the last basis along $f \omega$. Then $\left(b_{1}^{t}, 0\right), \ldots,\left(b_{n}^{t}, 0\right)$ represents the translation of $\gamma_{0}$ along $(f \omega, f \omega)$. On the other hand, $\gamma_{1}$ is represented by $\left(\ldots,\left(\left(f_{\#}-g_{\#}\right) a_{i}^{1}, 0\right), \ldots\right)$, which agrees with the above translation iff $\alpha_{1}$ agrees with the translation of $\alpha_{0}$. 
We will say that two points $x, y \in \Phi(f, g)$ are $R_{\mathrm{d}}$-related if there is a path establishing the Nielsen relation between them and for which one (hence each) of the conditions $\left(\mathrm{A}^{\prime \prime}\right),\left(\mathrm{B}^{\prime \prime}\right),\left(\mathrm{C}^{\prime \prime}\right)$ from $(2.1)$ holds. We notice that $\left(\mathrm{C}^{\prime \prime}\right)$ coincides with the reducibility relation from $[\mathrm{D}-\mathrm{J}]$. Now we are in a position to prove equality of $\mid$ ind $\left.\right|_{d}$ and $\mid$ ind $\left.\right|_{t}$. This will be done in the following theorem where we approximate the given pair by a pair simultaneously $\mathrm{d}$ - and t-transverse and use the version $(\mathrm{C})$ to prove the equality of the semi-indices in this case.

Theorem (2.3). Let $A$ be a Nielsen class of a $\Phi$-compact pair $f, g: M \rightarrow$ $N$. Then $\mid$ ind $\left.\right|_{\mathrm{t}}(f, g: A)=\mid$ ind $\left.\right|_{\mathrm{d}}(f, g: A)$.

P r o o f. Since both semi-indices are homotopy invariants we may assume that $f, g$ is a smooth d-transverse pair. Then $\Phi(f, g)=\left\{x_{1}, \ldots, x_{k}\right\}$ is finite. We will show that $f, g$ may be replaced by a pair which is simultaneously dand t-transverse. Fix euclidean neighbourhoods $U_{i}$ such that $f x_{i}=g x_{i}=$ $0 \in U_{i} \subset N(i=1, \ldots, k)$. Since d-transversality of $f, g$ means that the difference of the tangent maps $f_{*}-g_{*}$ is an isomorphism, by the Implicit Function Theorem there exist disjoint balls $U_{i}^{\prime}$ in $M$ such that $x_{i} \in U_{i}^{\prime}=$ $B\left(x_{i}, 2 \varepsilon_{i}\right) \subset M, g-f$ is a diffeomorphism on $U_{i}^{\prime}$ and $f U_{i}^{\prime} \cup g U_{i}^{\prime} \subset U_{i}$. Let $\eta_{i}: \mathbb{R} \rightarrow \mathbb{R}$ be a non-increasing smooth function satisfying $\eta_{i}\left(-\infty, \varepsilon_{i}\right]=1$, $\eta_{i}\left[2 \varepsilon_{i}, \infty\right)=0$.

Define

$$
\left(f^{\prime}, g^{\prime}\right)=\left\{\begin{array}{cc}
\left(\left(1-\eta_{i}\left(\left|x-x_{i}\right|\right)\right) f(x), g(x)-\eta_{i}\left(\left|x-x_{i}\right|\right) f(x)\right) & \text { for } x \in U_{i}^{\prime}, i=1, \ldots, k, \\
(f x, g x) & \text { otherwise. }
\end{array}\right.
$$

The pair $f^{\prime}, g^{\prime}$ is homotopic to $f, g, \Phi\left(f^{\prime}, g^{\prime}\right)=\Phi(f, g)$ and moreover $f^{\prime}, g^{\prime}$ is simultaneously t-transverse and d-transverse. It remains to show that for $f^{\prime}, g^{\prime}$ the relations $R_{\mathrm{t}}$ and $R_{\mathrm{d}}$ coincide. Until the end of this proof we will write $f, g$ instead of $f^{\prime}, g^{\prime}$. Let $x_{0}, x_{1} \in \Phi(f, g)$ and let a path $\omega$ establish the Nielsen relation between these points. Set $y_{i}=f x_{i}=g x_{i}(i=0,1)$ and fix an orientation of $M$ at $x_{0}: \gamma_{0} \in H_{n}\left(M, M-x_{0}\right) \simeq H_{n}\left(T_{x_{0}} M, T_{x_{0}} M-x_{0}\right)$. Then

$$
\left((f, g)_{*} \gamma_{0}\right)^{\mathrm{t}} \in H_{n}\left(y_{0} \times N, y_{0} \times\left(N-y_{0}\right)\right)
$$

and

$$
\begin{aligned}
& \left((f, g)_{*} \gamma_{0}\right)^{\mathrm{d}} \\
& \quad \in H_{n}\left((T(N \times N) / T(\Delta N))_{\left(y_{0}, y_{0}\right)},(T(N \times N)-T(\Delta N) / T(\Delta N))_{\left(y_{0}, y_{0}\right)}\right) \\
& \quad=H_{n}\left(T(N \times N)_{\left(y_{0}, y_{0}\right)},(T(N \times N)-T(\Delta N))_{\left(y_{0}, y_{0}\right)}\right) \\
& \quad=H_{n}\left(U_{0} \times U_{0}, U_{0} \times U_{0}-\Delta U_{0}\right)
\end{aligned}
$$


(where $U_{0}$ is a euclidean neighbourhood of $x_{0}$ ) are orientations of the microbundle $N \times N \rightarrow \Delta N$ and of the normal bundle $T(N \times N) / T(\Delta N)$ at $\left(y_{0}, y_{0}\right)$. Then the inclusion $y_{0} \times U_{0} \subset U_{0} \times U_{0}$ induces a homomorphism

$$
\begin{aligned}
H_{n}\left(y_{0} \times N, y_{0} \times\left(N-y_{0}\right)\right)=H_{n}\left(y_{0}\right. & \left.\times U_{0}, y_{0} \times\left(U_{0}-y_{0}\right)\right) \\
& \rightarrow H_{n}\left(U_{0} \times U_{0}, U_{0} \times U_{0}-\Delta U_{0}\right)
\end{aligned}
$$

sending $\left((f, g)_{*} \gamma_{0}\right)^{\mathrm{t}}$ to $\left((f, g)_{*} \gamma_{0}\right)^{\mathrm{d}}$. Let $\left(y_{s}, y_{s}\right)$ be a path in $\Delta N$ homotopic in $N \times N$ to $(f \omega, g \omega)$ and $U_{s}$ be the translation of $U_{0}$ along $\left(y_{s}, y_{s}\right)$. Then the translations of the above orientations $\left((f, g)_{*} \gamma_{0}\right)_{s}^{\mathrm{t}},\left((f, g)_{*} \gamma_{0}\right)_{s}^{\mathrm{d}}$ along $\left(y_{s}, y_{s}\right)$ are compatible under the maps induced by the inclusions $y_{s} \times U_{s} \subset U_{s} \times U_{s}$. On the other hand, let $\gamma_{1}$ be the translation of $\gamma_{0}$ along $\omega \subset M$. Then the orientations $\left((f, g)_{*} \gamma_{1}\right)^{\mathrm{t}},\left((f, g)_{*} \gamma_{1}\right)^{\mathrm{d}}$ (defined as for $\left.\gamma_{0}\right)$ correspond under the inclusion $y_{1} \times U_{1} \subset U_{1} \times U_{1}$, hence $\left(\left(f_{*}, g_{*}\right) \gamma_{0}\right)_{1}^{\mathrm{t}}=\left(\left(f_{*}, g_{*}\right) \gamma_{1}\right)^{\mathrm{t}}$ iff $\left(\left(f_{*}, g_{*}\right) \gamma_{0}\right)_{1}^{\mathrm{d}}=\left(\left(f_{*}, g_{*}\right) \gamma_{1}\right)^{\mathrm{d}}$. This means that the relations $R_{\mathrm{t}}$ and $R_{\mathrm{d}}$ coincide.

In particular, the Nielsen number introduced in Section 1 coincides, in the smooth case, with the number from [D-J].

3. A Wecken type theorem. We will show that the Nielsen number from Section 1 is the best lower bound on the number of coincidence points in dimensions $\geq 3$ (compare [Ji1], [D-K], [D-J]). We will follow the scheme from [Ji1].

Consider the following setting:

(*) $\quad P \subset W$ and $V$ are topological manifolds whose dimensions satisfy $p+v=w, p \geq 2, v \geq 3$, and $\xi$ is a normal microbundle of $P$ in $W$.

The crucial step is the folowing

Lemma (3.1) (A Whitney type lemma). Let $D$ denote a $v$-dimensional ball, $h: D \rightarrow W$ a map t-transverse to $\xi, h^{-1} P=\left\{x_{0}, x_{1}\right\}$ and suppose the points $x_{0}, x_{1}$ are $R$-related. Then $h$ is homotopic rel bd $D$ to a map into $W-P$.

Proof. We may assume that $h x_{0} \neq h x_{1}$ since otherwise we may compose $h$ near $x_{1}$ with a local isotopy of $W$ near $h x_{1}$. Let $\omega:[0,1] \rightarrow D$ be the straight line segment from $x_{0}$ to $x_{1}$. Then $h \omega$ is homotopic (rel ends) to a map $\omega^{\prime}$ into $P$. Since $\operatorname{dim} P \geq 2$ and $h x_{0} \neq h x_{1}$, we may assume that $\omega^{\prime}$ is a locally flat (hence flat) regular arc in $P$. Let $U$ denote a euclidean neighbourhood of $\omega^{\prime}$ in $W$.

We will show that $h$ is homotopic rel bd $D$ to a map $h^{\prime}$ satisfying $h^{\prime-1} P=$ $h^{-1} P=\left\{x_{0}, x_{1}\right\}$ and $h^{\prime}(\omega[0,1]) \subset U$. Then we take a euclidean neighbourhood $U_{0}$ of $\omega^{\prime} \subset P$ and we put $U=U_{0} \times \mathbb{R}^{d}$, a neighbourhood of the zero section of $\xi$ restricted to $U_{0} \subset P$. Since $h^{\prime}(\omega[0,1]) \subset U$, a euclidean neighbour- 
hood $D_{0}$ of $\omega[0,1] \subset D$ is also sent by $h^{\prime}$ into $U$. We consider the composition $\widehat{h}: D_{0} \stackrel{h^{\prime}}{\rightarrow} U=U_{0} \times \mathbb{R}^{d} \rightarrow \mathbb{R}^{d}$. The zeros of $\widehat{h}$ correspond to the elements of $h^{\prime-1} P$, hence $\widehat{h}^{-1}(0)=\left\{x_{0}, x_{1}\right\}$ and $\widehat{h}$ is t-transverse to $0 \subset \mathbb{R}^{d}$. The assumption $x_{0} R x_{1}$ implies that the degrees of $\widehat{h}$ at $x_{0}$ and $x_{1}$ are opposite, hence the induced homomorphism $\widehat{h}_{*}: H_{d}\left(D_{0}\right.$, bd $\left.D_{0}\right) \rightarrow H_{d}\left(\mathbb{R}^{d}, \mathbb{R}^{d}-0\right)$ is zero and by the Hopf lemma $\widehat{h}$ is homotopic rel bd $D_{0}$ to a map into $\mathbb{R}^{d}-0$. The last gives a homotopy rel bd $D_{0}$ from $h^{\prime}$ to a map into $W-P$.

It remains to construct $h^{\prime}$. Fix ball neighbourhoods $K_{i}$ of $x_{i} \in \operatorname{int} D$ so small that $h\left(K_{i}\right) \subset U$ and let $x_{i}^{\prime}=\omega\left(t_{i}\right)$ be the unique common point of $\omega[0,1]$ and bd $K_{i}(i=0,1)$.

The assumption that $h \omega$ is homotopic to $\omega^{\prime} \subset P$ implies a homotopy from $h \omega_{\mid\left[t_{0}, t_{1}\right]}$ rel $\left\{t_{0}, t_{1}\right\}$ to a map into $U$ (if only $K_{0}, K_{1}$ are small enough). Let $H:\left[t_{0}, t_{1}\right] \times[0,1] \rightarrow W$ denote this homotopy. We may assume that $H\left(\left(t_{0}, t_{1}\right) \times(0,1)\right) \cap P=\emptyset$ : we apply Lemma $(1.1)$ to $V=D=\left(t_{0}, t_{1}\right) \times(0,1)$, $C=\emptyset$ and

$$
\varepsilon(x, t)=\operatorname{dist}\left((x, t), \operatorname{bd}\left(\left[t_{0}, t_{1}\right] \times[0,1]\right)\right) .
$$

We may, moreover, assume that $H(t, 1) \notin P$ for any $t \in\left[t_{0}, t_{1}\right]$ (consider $H$ restricted to $\left[t_{0}, t_{1}\right] \times\left[0, s_{0}\right]$ for some $s_{0}$ close enough to 1$)$. Then we define a homotopy

$$
H^{\prime}: D \times 0 \cup\left(K_{0} \cup \omega\left[t_{0}, t_{1}\right] \cup K_{1}\right) \times I \rightarrow W
$$

by

$$
H^{\prime}(x, s)= \begin{cases}h(x) & \text { for } x \in K_{0} \cup K_{1} \text { or } s=0, \\ H(t, s) & \text { for } x=\omega(t), t \in\left[t_{0}, t_{1}\right] .\end{cases}
$$

Let $\varrho: D \times I \rightarrow D \times 0 \cup\left(K_{0} \cup \omega\left[t_{0}, t_{1}\right] \cup K_{1}\right) \times I$ be a retraction satisfying $\varrho^{-1}(x, t)=(x, t)$ for $x \in \operatorname{int}\left(K_{0} \cup K_{1}\right)$. Then the composition $H^{\prime \prime}=H^{\prime} \varrho: D \times I \rightarrow W$ satisfies $H^{\prime \prime}(x, 0)=h(x), H^{\prime \prime-1} P=\left\{x_{0}, x_{1}\right\} \times[0,1]$, $H^{\prime \prime}(\omega[0,1], 1) \subset U$. If $\delta: D \rightarrow[0,1]$ is an Urysohn function satisfying $\delta\left(\omega\left[t_{0}, t_{1}\right]\right)=1, \delta(\mathrm{bd} D)=0$ then $\bar{H}(x, t)=H^{\prime \prime}(x, \delta(x) t)$ is moreover a homotopy rel bd $D$. We put $h^{\prime}(x)=\bar{H}(x, 1)$.

Now following the Creating and Cancelling Procedures from [Ji1] we obtain

TheOREM (3.2) (A Wecken type theorem). Under the assumptions (*) any $\Phi$-compact map $h: V \rightarrow W$ is $\Phi$-compactly homotopic to a map $h^{\prime}$ so that $h^{\prime-1} P$ contains exactly $N(h)$ coincidence points.

We may assume that the homotopy from (3.2) has compact carrier, i.e. is constant outside a compact set. To see this let $h_{t}$ be a homotopy from (3.2) and let $\lambda: V \rightarrow[0,1]$ be a function equal to 1 on the compact set $\left\{x \in V: h_{t} x \in P\right.$ for some $\left.t \in[0,1]\right\}$ and to zero outside a compact set. We put $h_{t}^{\prime}=h_{\lambda(x) t}(x)$. 
Corollary (3.3). Let $f, g: M \rightarrow N$ be a $\Phi$-compact pair of maps between $n$-manifolds, $n \geq 3$. Then there is a homotopy $\left\{f_{t}\right\}$ starting from $f_{0}=f$, constant outside a compact set and such that $\Phi\left(f_{1}, g\right)$ contains exactly $N(f, g)$ elements.

Proof. We put in (3.2): $V=M, W=N \times N, h=(f, g), P=\Delta N, \xi=$ $(N \times N \ni(x, y) \rightarrow(x, x) \in \Delta N)$. We get a homotopy $\left(f_{t}, g_{t}\right)$ with compact carrier from $f, g$ to a pair with exactly $N(f, g)$ coincidence points. Now Theorem 1 of $[\mathrm{B}]$ yields a homotopy $f_{t}^{\prime}$ such that $f_{1}^{\prime}=f_{1}$ and $\# \Phi\left(f_{0}^{\prime}, g\right)=$ $N(f, g)$. It remains to show that the homotopy $f_{t}^{\prime}$ has a compact carrier. But we notice that if the carrier of the homotopy $g_{t}$ from the Lemma in Section 2 of $[\mathrm{B}]$ is compact then so is the carrier of the homotopy $h_{t}$ constructed there.

Finally, let us notice that all the results of [Je1] and [Je2] remain valid after replacing smooth manifolds by topological manifolds. In particular, we obtain a theorem expressing the Nielsen number of a fibre map by the Nielsen numbers of pairs between base spaces and fibres ([Je1], Thm. (4.3)) as well as a theorem expressing $N(f, g)$ by $N(\widetilde{f}, \widetilde{g})$ where $\widetilde{f}, \widetilde{g}$ are lifts to finite coverings ([Je2], Thm. (2.5)). These generalizations are evident, except possibly for Section 3 of [Je1]; but in fact the main result of that section (Thm. (3.13)) can be proved in a much simpler way in the TOP category.

\section{References}

[B] R. Brooks, On removing coincidences of two maps when only one rather than both of them may be deformed by a homotopy, Pacific J. Math. 40 (1972), 45-52.

[D-J] R. Dobreńko and J. Jezierski, The coincidence Nielsen theory on non-orientable manifolds, Rocky Mountain J. Math. 23 (1993), 67-85.

[D-K] R. Dobreńko and Z. Kucharski, On the generalization of the Nielsen number, Fund. Math. 134 (1990), 1-14.

[F] M. Freedman, The topology of four dimensional manifolds, J. Differential Geometry 17 (1982), 357-453.

[Je1] J. Jezierski, The semi-index product formula, Fund. Math. 140 (1992), 99-120.

[Je2] - , The coincidence Nielsen number for maps into real projective spaces, ibid., $121-136$.

[Ji1] B. J. Jiang, Fixed point classes from a differential viewpoint, in: Lecture Notes in Math. 886, Springer, 1981, 163-170.

[Ji2] - Lectures on the Nielsen Fixed Point Theory, Contemp. Math. 14, Amer. Math. Soc., Providence 1983.

[K-S] R. Kirby and L. Siebenmann, Foundational Essays on Topological Manifolds, Smoothings and Triangulations, Ann. of Math. Stud. 88, Princeton University Press, Princeton, 1977.

[Q] F. Quinn, Ends of maps III: Dimensions 4 and 5, J. Differential Geometry 17 (1982), 503-521. 
[Sc] M. Scharlemann, Transversality theories in dimension 4, Invent. Math. 33 (1976), 1-14.

[S] H. Schirmer, Mindestzahlen von Koinzidenzpunkten, J. Reine Angew. Math. 194 (1955), 21-39.

[V] J. Vick, Homology Theory, Academic Press, New York, 1973.

DEPARTMENT OF MATHEMATICS

UNIVERSITY OF AGRICULTURE

NOWOURSYNOWSKA 166

02-766 WARSZAWA, POLAND 